\title{
Efectos de un programa recreativo-pedagógico en las capacidades coordinativas en escolares
}

\section{Effects of a recreational-pedagogical program on coordinating capacities in school children}

\author{
Manuel Cárdenas ${ }^{1}$; Víctor Burbano ${ }^{2}$; Erwin Espitia ${ }^{3}$ \\ ${ }^{1}$ Licenciado en Educación Física, Recreación y Deportes, estudiante de Maestría en Pedagogía de la Cultura Física. Universidad Pedagógica y Tecnológica de \\ Colombia, UPTC. Tunja - Boyacá, Colombia, e-mail: manuel.cardenas@uptc.edu.co, Dhttps://orcid.org/0000-0003-4578-0357 \\ Licenciado en matemáticas, M.Sc. Ciencias-Estadística, Ph.D. Ciencias de la Educación. Universidad Pedagógica y Tecnológica de Colombia-UPTC, Grupo \\ de investigación GICl, e-mail: victor.burbano@uptc.edu.co, Dhttps://orcid.org/0000-0002-3561-1886 \\ ${ }^{3}$ Licenciado en Educación Física, Recreación y Deportes, M.Sc. Pedagogía de la Cultura Física. Colegio Liceo Moderno Celestín Freinet, Yopal, Casanare, \\ Colombia, e-mail: erwinyesidpamplona@gmail.com, Dhttps://orcid.org/0000-0001-9830-3664 \\ Cómo citar: Cárdenas, M.; Burbano, V.; Espitia, E. 2019. Efectos de un programa recreativo-pedagógico en las capacidades coordinativas \\ en escolares. Rev. U.D.C.A Act. \& Div. Cient. 22(1):e1047. https://doi.org/10.31910/rudca.v22.n1.2019.1047. \\ Artículo de acceso abierto publicado por Revista U.D.C.A Actualidad \& Divulgación Científica bajo una licencia Creative Commons \\ CC BY-NC 4.0 \\ Recibido: Octubre 23 de 2018 \\ Aceptado: Mayo 17 de 2019
}

\section{RESUMEN}

Los bajos niveles de coordinación diagnosticados en los niños pueden afectar su desarrollo motriz posterior y su rendimiento académico, por lo tanto, se justifica formular y evaluar programas de intervención, que mejoren sus capacidades coordinativas. El objetivo de este estudio fue determinar el efecto de un programa recreativo pedagógico sobre las capacidades coordinativas, de 64 estudiantes, tanto de género masculino como femenino, con edades entre los 6 y 7 años, en una Institución educativa colombiana. En la investigación efectuada, se utilizó una metodología de corte cuantitativo, con un diseño cuasi experimental-descriptivo; la información, se recolectó mediante el test de Capón, usado antes y después de aplicar el programa recreativo-pedagógico diseñado. El procesamiento de los datos, se efectuó con el software libre R. Los resultados permitieron establecer que, en promedio, las diferencias entre las puntuaciones acumuladas entre el pre-test y el pos-test en el grupo experimental, sí son significativas y atribuibles al mencionado programa. En conclusión, tal programa sí produce efectos positivos en las capacidades coordinativas de los participantes en el estudio.
Palabras clave: coordinación; infantil; actividades lúdicas; educación física; programa pedagógico.

\section{ABSTRACT}

The low levels of coordination diagnosed in children can affect their subsequent motor development and their academic performance; therefore, it is justified to formulate and evaluate intervention programs that improve their coordinative capacities. The objective of this study was to determine the effect of a pedagogical recreational program on the coordinative abilities of 64 male and female students aged between 6 and 7 years in a Colombian educational institution. In the research carried out, a quantitative cut-off methodology with a quasi-experimental-descriptive design was used; the information was collected by means of the Capón test, used before and after applying the designed recreational-pedagogical program. The processing of the data was done with free software $\mathrm{R}$. The results allowed to establish that, on average, the differences between the scores accumulated between the pre-test and the post-test in the experimental group are significant and attributable to the aforemen- 
tioned program. In conclusion, such a program does have positive effects on the coordinative abilities of the study participants.

Keywords: coordination; children; play activities; physical education; pedagogical program.

\section{INTRODUCCION}

El cuerpo del niño entre los seis y siete años, se encuentra en pleno crecimiento y el profesor de educación física, se constituye en un elemento fundamental, para potenciar el desarrollo de sus capacidades coordinativas. Mediante formación permanente e intervención oportuna puede ayudarlo a que mejore sus movimientos y destrezas corporales, de modo que incremente sus competencias físicas, sociales, comunicativas y académicas (Martínez et al. 2019). En este contexto, el diseño y la aplicación de un programa recreativo-pedagógico puede favorecer el desarrollo de dichas capacidades; además, cuando el docente conoce los niveles de "coordinación motriz" de los niños puede planear, adoptar protocolos y generar estrategias de intervención, tanto individuales como colectivas, que puedan posibilitar un mejor rendimiento motriz y académico en sus estudiantes (Vidarte et al. 2018).

Adicionalmente, las capacidades coordinativas son requeridas por el ser humano, para cumplir múltiples tareas en su vida diaria; en este sentido, la coordinación motriz corresponde al conjunto de capacidades tendientes a organizar y regular los procesos parciales de una acción motora, en función de un objetivo propuesto con antelación (Cenizo Benjumea et al. 2017). La literatura reporta la existencia de seis capacidades: i) equilibrio, ii) orientación iii) reacción, iv) diferenciación, v) combinación y vi) adaptación y modificación; algunos autores mencionados en Caminero (2006), agregan una séptima, denominada capacidad de ritmo y, en Jacob (1991), se propone una adicional llamada capacidad de asociación. Estas capacidades, se pueden potenciar con la ejecución de actividades asociadas con el esquema corporal, saltos, equilibrio, coordinación óculo-manual y desplazamientos. En seguida, se describen estos aspectos, que forman parte del marco teórico.

El 'esquema corporal' está referido a una organización global de carácter psicomotriz, que abarca: mecanismos, procesos motores con niveles perceptivos, tónicos y sensoriales; en éstos, el nivel afectivo, frecuentemente, se encuentra inmerso, tanto en circunstancias de movimiento o de quietud. Los saltos corresponden al despegue respecto de una superficie determinada, que se puede generar con carrera previa o sin ella; además, incluye un lapso de suspensión y un retorno (caída) del individuo hacia tal superficie; también, puede incluir la dinámica de una o ambas piernas. El equilibrio o control postural es un estado, donde las fuerzas actuantes sobre el cuerpo de un determinado individuo se compensan, de tal modo, que el cuerpo logra mantenerse en una posición de interés. El equilibrio es esencial para la adquisición de habilidades motoras, el desarrollo de las actividades diarias del niño y la maduración del sistema nervioso central (Ebrahimi et al. 2017).
La coordinación óculo-manual, también denominada viso-motriz, corresponde a la capacidad que posee el niño para coordinar su vista con movimientos específicos de su cuerpo o con otras partes del mismo. Esta coordinación juega un rol importante, en el sentido que, en ella, se conjugan la motricidad fina de las manos con el campo visual del individuo, requeridas para potenciar el aprendizaje de la escritura y la lectura, entre otros. Finalmente, el desplazamiento se conceptualiza como la progresión realizada entre dos puntos en un espacio especificado, mediante el movimiento del cuerpo y corresponde a una de las 'habilidades motrices básicas', requeridas en la escuela (Prieto, 2010).

Aunque, de acuerdo con la Constitución de Colombia, todos los nacidos en este país, sin discriminación por edad, sexo o raza, tienen derecho a la práctica de diversas actividades que promuevan el desarrollo sano del ser humano y una mejora en la calidad de vida, ya sea mediante la intervención estatal directa o a través de las instituciones educativas (Ramos, 2018), en general, se presentan dificultades para entender y aplicar las capacidades coordinativas en actividades recreativas o en la clase de educación física (Caminero, 2006). En este sentido, Carrasco \& Torres (1998) aseguran que se debe prestar atención a los factores que generan falencias, tales como: i) la edad, ii) el grado de fatiga, iii) la condición física, iv) el nivel de aprendizaje, v) la tensión nerviosa, vi) la zona del cuerpo (manos o pies), vii) la intensidad del esfuerzo, viii) la destreza de los miembros superiores y ix) la dirección y sentido del movimiento.

Por otro lado, un elemento requerido para potenciar las capacidades coordinativas es la lúdica, como parte de los programas recreativos, que se constituye en uno de los factores que motiva a los niños y favorece la realización de clases divertidas. Al respecto, en Sánchez (2015), se indica que la lúdica es necesaria para el crecimiento personal, que lleva a la búsqueda del sentido de la vida, la fantasía, la creatividad y la libertad. Por otro lado, el juego también se puede incluir en los programas recreativos, a fin de promover el desarrollo de las capacidades coordinativas; según Raabe (1980), el juego se puede entender como una actividad o acción voluntaria, que se realiza bajo ciertos límites prefijados, tanto en el tiempo como en un lugar, en correspondencia con reglas aceptadas de forma libre, pero provista de un fin en sí misma; se acompaña de alegría, de una conciencia por ser de otra manera en la vida diaria y matizada con un determinado sentimiento.

Por otra parte, el test de Capón, adaptado por Carrasco (1998), puede ser utilizado por profesores y por entrenadores, para diseñar e implementar programas que favorezcan el progreso de los niños, en el desarrollo de sus capacidades coordinativas, necesarias para mejorar su calidad de vida, su academia y sus actividades deportivas. Este test es un instrumento que evalúa el grado de desarrollo motriz en niños desde los 4 y hasta los 10 años de edad, valora las áreas de conocimiento corporal, espacial, equilibrio, coordinación general y coordinación óculo manual. El test tiene una valoración mínima de 6 puntos y una máxima de 24 puntos, que indica el nivel o grado de tal desarrollo en el niño. En el test, se incluyen seis tareas: 
i) identificación de las partes del cuerpo, ii) tabla de equilibrio, iii) salto con un pie, iv) salto y caída v) recorrido con obstáculos y vi) recepción de un balón. En este contexto, Leiva et al. (2015) usaron este test con niños de 7 y 8 años, para comparar tales capacidades, en dos grupos de estudio. Asimismo, Ardila Muñoz et al. (2017), lo emplearon con niños en edad escolar, con el propósito de establecer las existencias de mejoras significativas después de aplicar un programa alusivo a las capacidades coordinativas. Estudios, como los mencionados, indican que sí es posible que el docente formule y evalúe programas específicos de intervención, destinados a la mejora de tales capacidades.

En estas circunstancias, el objetivo de esta reflexión fue determinar el efecto de un conjunto de actividades recreativas sobre las capacidades coordinativas de los niños participantes. En el logro de tal objetivo, el docente de educación física se ha de constituir en un elemento clave, puesto que, amparado en la legislación colombiana, puede promover la actividad física en la escuela, de modo que favorezca el desarrollo integral del niño, mejore sus capacidades al interactuar con programas recreativos, se diagnostique el estado de la motricidad y la coordinación de los estudiantes que empiezan su etapa escolar primaria y evalúe el efecto de un programa en específico sobre tales capacidades, de manera que se generen nuevas reflexiones y recomendaciones focalizadas en su implementación.

\section{MATERIALES Y MÉTODOS}

En este trabajo, se utilizó un enfoque de investigación empírico-analítico, de tipo cuantitativo y se usó un diseño cuasi-experimental, se trabajó con un grupo experimental y un grupo control. La muestra fue seleccionada a conveniencia y se conformó con 64 estudiantes, de grado primero de educación básica primaria, con edades de 6 y 7 años, pertenecientes a la Institución Educativa San Jerónimo Emiliani, en Tunja-Boyacá, en Colombia. Se decidió hacer un muestreo por conveniencia, teniendo en cuenta las características del test de Capón y los criterios de inclusión, tales como: que el niño estuviera matriculado en el grado primero y en capacidad de realizar actividad física en consonancia con su edad, se excluyeron quienes presentaban algún impedimento físico o no tuvieran el consentimiento de sus padres.

En cuanto al género, en el grupo control, 8 estudiantes (25\%) eran de género femenino y 24 (75\%) masculino; en el grupo experimental, 14 participantes $(43,8 \%)$ fueron de género femenino y $18(56,3 \%)$ masculino; estos porcentajes difieren levemente con los obtenidos por Torralba et al. (2014). La información fue recolectada, a través del instrumento denominado test de Capón, que permite medir las capacidades coordinativas en cinco niveles: con problemas, regular, aceptable, bueno y muy bueno, en correspondencia con las puntuaciones que obtenga el niño; este instrumento, se utilizó antes y después de aplicar el programa recreativo-pedagógico a los participantes. Referente a la participación de los niños, en el 2017, los padres de familia o acudientes firmaron un consentimiento informado. La hipótesis del estudio estuvo centrada en probar si un programa recreativo-pedagógico de 12 semanas influía positivamente en el desarrollo de las capacidades coordinativas en tales niños.
El programa recreativo pedagógico consistió en realizar actividades, ejercicios, juegos y rondas infantiles en correspondencia con las seis tareas propuestas en el test de Capón y se llevó a cabo en un periodo de 12 semanas, tres días por cada semana, con una intensidad de una hora por día; este programa inició el 31 de julio de 2017 y finalizó el 19 de octubre, del mismo año. En cada semana, se trabajó una tarea; en consecuencia, al empezar la séptima semana, se repite nuevamente la primera tarea. Cada clase inicia con ejercicios de calentamiento, que incluyen rondas infantiles. En la primera semana, se hicieron ejercicios y juegos destinados a potenciar el conocimiento del cuerpo de cada niño; en la segunda, se trabajaron ejercicios sobre equilibrio, entre ellos, equilibrio sobre una tabla y las líneas de la cancha multifuncional de la Institución Educativa; en la tercera semana, se ejecutaron saltos con un pie; los ejercicios implementados fueron con un pie y posteriormente con el otro, siempre cambiando el material, ya sea con lasos, aros, tablas, entre otros; en la cuarta, se hizo salto y caída; los ejercicios se realizaron con un cajón, sillas, lasos y elementos del entorno; en esta tarea, se combinó la carrera, el salto y la caída (el niño corre, se sube al cajón y luego cae); en la quinta semana, se hace un recorrido con obstáculos, donde los ejercicios elaborados se desarrollaron esquivando implementos, tales como sillas, balones, aros, lasos, entre otros y en la sexta, se hacen actividades que incluye la recepción de un balón con las manos; los ejercicios elaborados fueron con un balón trabajando tanto individual como grupalmente; asimismo, se lanzó un balón hacia arriba o hacia el frente (se trabajó en grupo grande o por parejas). Para cada estudiante, se llevó una planilla de registro sobre las puntuaciones logradas por el niño, en concordancia con el test de Capón. Para el análisis de datos, se utilizó el software libre $\mathrm{R}$ y se calcularon algunas medidas estadísticas, como porcentajes, promedios, desviación estándar (DS) y coeficiente de variación (CV), que permiten interpretar los resultados. Cuando el CV es inferior al $8 \%$, los individuos son similares en la característica cuantitativa de interés; si el CV está entre el 8 y el 18\% son casi similares; si el coeficiente de variación es superior al 18\%, los individuos no son parecidos (Burbano \& Valdivieso, 2016).

\section{RESULTADOS Y DISCUSIÓN}

En esta sección, se presentan los resultados obtenidos y se hace una discusión de los mismos. Inicialmente, se hace una breve caracterización de los individuos que participaron en el estudio; se prosigue con un diagnóstico sobre los niveles de clasificación de los participantes respecto de sus capacidades coordinativas, tanto en el grupo control como en el experimental en el pre-test; se continúa con los resultados de la aplicación del programa recreativo-pedagógico (pos-test) y, por último, se prueban las hipótesis planteadas y se generan las conclusiones.

Caracterización de los participantes por edad, talla y peso. El grupo control, se conformó por 32 individuos, con edades entre los 6 y 7 años, distribuidos de la siguiente forma: 17 niños $(53,1 \%)$, con edad de 6 años y 15 (46,9\%), con 7 años de edad; el promedio de edad fue de 6,47 $\pm 0,507$ años, con DS $\pm 0,507$ y un CV $=0,0783$ $=7,83 \%$. El grupo experimental, se constituyó por 32 individuos, con edades entre los 6 y 7 años, distribuidos así: 12 niños (37,5\%), 
con 6 años y 20 (62,5\%), con 7 años de edad; el promedio fue de 6,63 años, con DS $\pm 0,492$ y un $\mathrm{CV}=0,0742=7,42 \%$. El CV obtenido indica que los participantes resultaron con similar edad en ambos grupos.

Respecto a la talla, en el grupo control, se obtuvo un promedio de 120,09 centímetros, con DS $\pm 3,872$ y un $C V=0,0322=3,22 \%$; en el grupo experimental, se determinó un promedio de $120,09 \mathrm{~cm}$, con DS \pm 4,439 y un CV $=0,0369=3,69 \%$; el CV resultante indica que los participantes presentaron tallas semejantes en ambos grupos. Referente al peso, en el grupo control, el promedio fue de 23,44 kilogramos, con DS $\pm 2,996$ y un $C V=0,1278=12,78 \%$; en el grupo experimental, el promedio fue de 23,98 kilogramos, con DS $\pm 3,114$ y un $C V=0,1298=12,98 \%$; el CV indica que los niños fueron casi parecidos respecto al peso. Caracterizaciones semejantes se han realizado en otros estudios en contextos similares; Leiva et al. (2015) desarrollaron su estudio con 80 participantes, con edades entre 7 y 8 años, relativamente similares en cuanto a la edad (CV= $0,077=7,7 \%$, la talla y el peso.

Adicionalmente, se obtuvo el Índice de Masa Corporal (IMC), encontrándose los siguientes resultados: i) En el grupo control, el IMC varía entre un mínimo de 13,67 y un máximo de $21,12 \mathrm{~kg} / \mathrm{m}^{2}$, el promedio fue de $16,2146 \pm 1,45461 \mathrm{~kg} / \mathrm{m}^{2}$, con DS $\pm 1,45461 \mathrm{y}$ un $\mathrm{CV}=0,0897=8,97 \%$; ii) En el grupo experimental, el IMC tiene un mínimo de 12,77 y un máximo de $20,94 \mathrm{~kg} / \mathrm{m}^{2}$, el promedio fue de $16,6023 \pm 1,66202 \mathrm{~kg} / \mathrm{m}^{2}$, con DS $\pm 1,66202$ y un $\mathrm{CV}=0,1001$ $=10,01 \%$. El CV obtenido indica que los niños de ambos grupos resultaron casi parecidos respecto a su IMC (Tabla 1).

Diagnóstico sobre los niveles de clasificación en las capacidades coordinativas. En concordancia con el test de Capón, se establecen los siguientes niveles para las capacidades coordinativas: i) "con problemas", cuando el individuo obtiene 12 o menos de puntuación acumulada; ii) "regular", de 13 a 15 puntos; iii) "aceptable", de 16 a 18 puntos; iv) "bueno", de 19 a 21 puntos y v) "muy bueno”, de 22 a 24 puntos.

En el pre-test, los participantes del grupo control se distribuyeron así: $12(37,5 \%)$ con problemas, $14(43,8 \%)$ en el nivel regular, 4 $(12,5 \%)$ en aceptable y $2(6,3 \%)$ en bueno; en el grupo experimental, $9(28,1 \%)$ con problemas, $21(65,6 \%)$ en el nivel regular y $2(6,3 \%)$ en aceptable. Estos resultados permiten interpretar que los estudiantes del grupo experimental presentan una tendencia a ubicarse en un alto porcentaje (93,7\% acumulado) en los niveles con problemas y regular, en sus capacidades coordinativas; estas deficiencias son comparables con las diagnosticadas en el estudio realizado por Ardila et al. (2017), quienes encontraron que el 68,3\% de los individuos se ubicaron en la categoría "con problemas".

Adicionalmente, en el grupo control, el promedio de las puntuaciones acumuladas fue de 13,59 , con DS $\pm 2,710$ y un $C V=0,1994=$ $19,94 \%$; con base en estos resultados, de manera general, el grupo control se clasifica en la categoría de "regular" y los individuos resultaron casi parecidos respecto a tales puntuaciones. En el grupo experimental, el promedio de las puntuaciones acumuladas fue de 13,09 , con DS $\pm 1,973$ y un $C V=0,1507=15,07 \%$; de forma general, el grupo experimental también se ubica en la categoría de "regular" y los individuos fueron casi similares.

Aplicación del programa recreativo-pedagógico. Después de aplicar el programa recreativo-pedagógico de capacidades coordinativas en el grupo experimental (pos-test) y no de aplicarlo en el grupo control, se determinó que, en el pos-test, los participantes del grupo control se distribuyeron así: 7 (21,9\%) con problemas, $6(18,8 \%)$ en el nivel regular, $12(37,5 \%)$ en aceptable, $5(15,6 \%)$ en bueno y 2 $(6,3 \%)$ en muy bueno; en el grupo experimental, $19(59,4 \%)$ en el nivel bueno y $13(40,6 \%)$ en muy bueno. Estos resultados permiten interpretar que los estudiantes del grupo experimental mejoraron sus puntuaciones acumuladas, de manera que se ubicaron en los niveles bueno y muy bueno, es decir, la aplicación del programa ha generado efectos positivos en los participantes.

Estas mejoras son relativamente superiores a las obtenidas en el estudio elaborado por Ardila et al. (2017), quienes encontraron que el 93,3\% de los individuos, se ubicaron en las categorías "bueno" y "muy bueno". De manera similar, Leiva et al. (2015) reportan que los individuos participantes en su estudio, se clasificaron en la categoría “con problemas" (20,6\%), "regular" (14,7\%), "aceptable" (41,2\%), "bueno" (14,7\%) y "muy bueno" (8,8\%); estos porcentajes permiten establecer que solamente el 23,5\% se clasificaron en las categorías de "bueno" y "muy bueno".

Además, en el grupo control, el promedio de las puntuaciones acumuladas fue de 16,16 , con DS $\pm 3,629$ y un $C V=0,2245=22,45 \%$; teniendo en cuenta estos resultados, de manera general, el grupo control se clasifica en la categoría de "aceptable" y los individuos resultaron poco similares respecto a tales puntuaciones. En el grupo experimental, el promedio de las puntuaciones acumuladas fue de 21,28 , con DS $\pm 1,631$ y un $C V=0,0766=7,66 \%$; teniendo

Tabla 1. Índice de Masa Corporal (IMC) de los niños participantes en el estudio.

\begin{tabular}{|l|c|c|}
\hline \multicolumn{1}{|c|}{ Grupo } & Control & Experimental \\
\hline Promedio & 16,2146 & 16,6023 \\
\hline Desviación estándar & 1,45461 & 1,66202 \\
\hline Mínimo & 13,67 & 12,77 \\
\hline Máximo & 21,12 & 20,94 \\
\hline
\end{tabular}


en cuenta estos resultados, de manera general, el grupo control se clasifica en la categoría de "muy bueno" y los individuos fueron casi similares. Estos resultados descriptivos permiten interpretar que, como consecuencia de la aplicación del programa, se producen considerables diferencias promedio en las puntuaciones acumuladas y en la similitud de los individuos del grupo experimental con respecto al grupo control, en referencia a sus capacidades coordinativas.

Prueba de hipótesis. A continuación, se efectúa un análisis de carácter inferencial, el cual, permite establecer si existen diferencias significativas entre las puntuaciones acumuladas logradas por los niños en el pos-test en comparación con las obtenidas en el pre-test, para el grupo experimental. Para desarrollar este proceso de inferencia estadística, se calcularon porcentajes de mejora y se efectuaron pruebas estadísticas. Finalmente, se establece que el porcentaje de mejora del grupo experimental es significativamente mayor al del grupo control. En la prueba de hipótesis, se utiliza un nivel de significancia del 0,05 (5\%).

Las hipótesis planteadas en el estudio fueron:

$\mathrm{H}_{0}$ : El programa recreativo pedagógico no influye sobre las capacidades coordinativas de los niños de 6-7 años de la Institución educativa San Jerónimo Emiliani, en Tunja, Boyacá.

$\mathrm{H}_{1}$ : El programa recreativo pedagógico sí influye sobre las capacidades coordinativas de los niños, quienes participaron en este estudio.

En primera instancia, se obtuvieron las puntuaciones promedio, tanto para el pre-test como para el pos-test en el grupo experimental. Se estableció que la puntuación promedio del pos-test había aumentado considerablemente, al pasar de 13,09 a 21,28, lo que indica que se generó un porcentaje promedio de mejora aproximado, de 62,56\%, en el grupo experimental, como consecuencia de la aplicación del programa recreativo-pedagógico. En esta dirección, en el estudio de Ardila et al. (2017), se obtuvo un promedio de 22,17, para grado cuarto y de 22,38, para el quinto, de Educación básica primaria en Colombia. El promedio, levemente inferior logrado por los individuos del presente estudio, se puede atribuir a que los niños presentan edades inferiores a los del trabajo realizado por Ardila et al. (2017).

Luego, se estableció, mediante la prueba K-S (de Kolmogorov-Smirnov al $5 \%$ ), que los datos de las puntuaciones acumuladas, tanto del pre-test (P-valor 0,155) como del pos-test (P-valor 0,338), se ajustaban a una distribución normal; en consecuencia, se aplicó una prueba t-student para muestras pareadas, con un nivel de significancia del 0,05; el P-valor obtenido en la prueba (Sig. 2-tailed) fue de 0,000 , el cual, es inferior a 0,05 , por lo tanto, se tiene que, en promedio, las diferencias entre las puntuaciones acumuladas entre el pos-test y el pre-test en el grupo experimental, sí son significativas. En consecuencia, sus respectivos porcentajes de mejora también son significativos y se atribuyen a la aplicación del programa recreativo-pedagógico. En conclusión, sí se produjeron incrementos significativos en las puntuaciones acumuladas de algunos niños del grupo experimental, que implican un efecto positivo en los porcentajes de mejora respecto de las capacidades coordinativas.

Finalmente, se decidió comparar los promedios de mejora por grupos. Se determinó que el porcentaje de mejora promedio en el grupo experimental fue del $62,56 \%$; este porcentaje fue superior al del grupo control, de 23,45\%, lo cual, proporciona evidencias de que el programa recreativo-pedagógico ha surtido efectos positivos sobre las capacidades coordinativas de los niños que pertenecen al grupo experimental.

Además, una vez se verificó que los porcentajes de mejora, tanto en el grupo control (promedio 23,45\%) como experimental (promedio $66,89 \%$ ), superaron la prueba de normalidad, con un P-valor de 0,496 y de 0,394 , respectivamente (Tabla 2); se aplicó una prueba t-student para muestras independientes, con un nivel de significancia del 0,05 (5\%). El P-valor de la prueba (Sig. 2-tailed) fue de 0,000, que es inferior a 0,05 (Tabla 3), por lo tanto, se tiene que, en promedio, las diferencias entre los porcentajes de mejora correspondientes al grupo experimental y al grupo control sí son significativas. Por ende, los porcentajes de mejora del grupo experimental son significativamente mayores a los porcentajes del grupo control. Estos porcentajes de mejora en el grupo experimental, se atribuyen a la aplicación del programa recreativo-pedagógico. En síntesis, los porcentajes de mejora del grupo experimental son significativamente superiores a los del grupo control; en este contexto, el programa ha generado efectos positivos en las capacidades coordinativas de los niños, que conformaron el grupo experimental.

Así, entonces, la disposición de los niños y las actividades implementadas, se constituyen en motores potenciales para el mejoramiento de las capacidades coordinativas de los niños pertenecientes al

Tabla 2. Prueba K-S para los porcentajes de mejora.

\begin{tabular}{|l|r|r|}
\hline & \multicolumn{1}{|c|}{$\begin{array}{c}\text { Porcentaje de mejora } \\
\text { Grupo Control }\end{array}$} & \multicolumn{2}{|c|}{$\begin{array}{c}\text { Porcentaje de mejora } \\
\text { Grupo Experimental }\end{array}$} \\
\hline $\mathbf{N}$ & $\mathbf{3 2}$ & $\mathbf{3 2}$ \\
\hline Promedio & $23,45 \%$ & $\mathbf{6 6 , 8 9 \%}$ \\
\hline Desviación estándar & 37,19 & 33,66 \\
\hline Kolmogorov_smirnov Z & 0,83 & 0,899 \\
\hline Significancia bilateral & 0,496 & 0,394 \\
\hline
\end{tabular}


Tabla 3. Prueba t-student para muestras independientes.

\begin{tabular}{|l|c|c|c|c|c|}
\hline \multicolumn{1}{|c|}{ Experim. vs Contr. } & $\begin{array}{c}\text { Test de } \\
\mathbf{F}\end{array}$ & $\begin{array}{c}\text { Levene } \\
\text { Sig. }\end{array}$ & $\begin{array}{c}\text { t-test } \\
\mathbf{t}\end{array}$ & $\begin{array}{c}\text { Grados } \\
\mathbf{d f}\end{array}$ & $\begin{array}{c}\text { Sig-2 } \\
\text { Tailed }\end{array}$ \\
\hline Varianzas iguales & 1,253 & 0,267 & 4,899 & 62 & 0,000 \\
\hline Varianzas desiguales & & & 4,899 & 61,393 & 0,000 \\
\hline
\end{tabular}

grupo experimental, bajo el tutelaje del docente, quien ha orientado a los niños en estas actividades. Lo anterior guarda relación con el estudio realizado por Vidarte \& Orozco (2015), quienes encontraron una correlación significativa entre la coordinación motriz y el rendimiento académico manifestado, a través de las dimensiones cognitiva, afectiva, comunicativa, corporal, ética y estética; además, indican que el docente ha de promover la actividad física, de modo que favorezcan el desarrollo integral del niño, atendiendo a sus características particulares; para esto, se requiere efectuar diagnósticos, que posibiliten determinar el estado de los aspectos asociados con la motivación, la motricidad y la coordinación del niño.

Con base en el análisis descriptivo efectuado sobre los datos del pretest, se concluye que los niños pertenecientes, tanto al grupo control como al grupo experimental, se clasificaron, de manera grupal, en la categoría "regular", al inicio del presente trabajo investigativo. Además, como el coeficiente de variación obtenido indica que, tanto los niños del grupo control como del grupo experimental son casi similares en cuanto a las puntuaciones acumuladas obtenidas a través del test aplicado, este trabajo investigativo arrancó con grupos similares, en cuanto a su estado de desarrollo de sus capacidades coordinativas, lo que permitió determinar el efecto del programa recreativo pedagógico.

El análisis descriptivo efectuado con los resultados del pos-test permitió concluir que los niños pertenecientes al grupo control se clasificaron, de forma grupal, en la categoría "aceptable", mientras que los niños del grupo experimental lograron ubicarse en la categoría de "bueno". Mediante el coeficiente de variación obtenido, se estableció que los niños del grupo experimental resultaron parejos en el pos-test, en cambio, los niños del grupo control, fueron disparejos, en cuanto a las puntuaciones acumuladas obtenidas; por consiguiente, el trabajo investigativo permite percibir considerables diferencias promedio en las puntuaciones acumuladas asociadas con el desarrollo de sus correspondientes niveles en sus capacidades coordinativas.

Del análisis inferencial, se concluye que, en promedio, las diferencias entre las puntuaciones acumuladas entre el pos-test y el pre-test en el grupo control, sí son significativas, a pesar de no haberse aplicado el programa. Asimismo, se deduce que, en promedio, las diferencias entre las puntuaciones acumuladas entre el pos-test y el pre-test, en el grupo experimental, sí son significativas y atribuibles al programa recreativo pedagógico.

Finalmente, se concluye que, en promedio, existen diferencias significativas entre los porcentajes de mejora correspondientes al grupo experimental y al grupo control, es decir, los porcentajes de mejora del grupo experimental son significativamente mayores a los porcentajes del grupo control y se atribuyen a la aplicación del programa recreativo pedagógico. En estas circunstancias, tal programa produce efectos positivos en las capacidades coordinativas de los niños del grupo experimental. Se recomienda a los docentes que trabajan con niños en edades tempranas, que apliquen programas recreativos con actividades similares a las aquí planteadas, a fin de mejorar las capacidades coordinativas de los niños, motiven a los infantes para que participen activamente en la clase de educación física y planifiquen actividades recreativas acordes a la edad, su desarrollo coordinativo e intereses de los niños, antes de ofrecer sus clases de educación física.

Agradecimientos y financiación. Este artículo se deriva de un proyecto de investigación desarrollado al interior del programa de Maestría en Pedagogía de la Cultura Física de la Universidad Pedagógica y Tecnológica de Colombia. Conflictos de intereses. Este escrito fue elaborado y luego revisado por cada uno de los autores, quienes manifiestan la inexistencia de conflicto de intereses, que ponga en riesgo la validez de los resultados, que se presentan en este documento.

\section{REFERENCIAS}

1. ARDILA MUÑOZ, A.E.; MELGAREJO PINTO, V.M.; GALINDO, D. 2017. Incidencia de un programa de ejercicios físicos sobre las capacidades coordinativas en población escolar. Rev. Salud, Historia y Sanidad. 12(1):133-148. https://doi.org/10.1909/shs.v12i1.174

2. BURBANO, V.; VALDIVIESO, M. 2016. Inferencia estadística básica, apoyo al estudio independiente. Editorial UPTC. Tunja, Colombia. 222p.

3. CENIZO BENJUMEA, J.; RAVELO AFONSO, J.; MORILLA PINEDA, S.; FERNÁNDEZ TRUAN, J.C. 2017. Test de coordinación motriz 3JS: Cómo valorar y analizar su ejecución. Retos: nuevas tendencias en educación física, deporte y recreación. Retos. 32(2):189-193.

4. CAMINERO, F.L. 2006. Marco teórico sobre la coordinación motriz. Lecturas: Educación física y deportes. 93(1):17-24.

5. CARRASCO, S. 1998. Un enfoque psicomotor, Programa de educación física para alumnos de primero y segundo año de Educación básica. Inter-Gráfica Ltda (Chile). 128p. 
6. CARRASCO, L.; TORRES, G. 1998. La coordinación como factor principal en la enseñanza del tenis: aplicación en la escuela. Rev. Ciencias de la Actividad Física y del Deporte. 12(1):41-46.

7. EBRAHIMI, A.A.; MOVALLALI, G.; JAMSHIDI, A.A.; RAHGOZAR, M.; HAGHGOO, H.A. 2017. Postural control in deaf children. Act. Med. Iranica. 55(2):115-122.

8. JACOB, F. 1991. Función e importancia de las cualidades coordinativas. Rev. Stadium. 25(147):36-40.

9. LEIVA, V.M.; ALVARADO, V.Ch.; GALLARDO, R.R.; VARGAS, V.R.; MARTÍNEZ, S.C.; CARRASCO, A.V. 2015. Desarrollo motor en escolares con diferentes aprestos formativos motrices. Rev. Ciencias de la Actividad Física UCM. 16(1):19-28.

10. MARTÍNEZ, P.Y.; LÓPEZ, J.A.; LÓPEZ, A.A.; CASTRO, Z.E.; BUÑUEL, P.S.; GARCÍA, C.C. 2019. Análisis comparativo de un programa de educación física en niños con discapacidad auditiva sobre edad motora equivalente. Retos. 35(1):310-313.

11. PRIETO, M. 2010. Habilidades motrices básicas. Innovaciones y experiencias educativas, 10 . Disponible desde Internet en: http://jorgegarciagomez.org/documentos/hmbasicas.pdf (con acceso 15/04/2017).
12. RAABE, J. 1980. El niño y el juego. Planteamientos teóricos y aplicaciones pedagógicas. Rev. Trimestral de Educación. 34(1):5-23.

13. RAMOS, A.J. 2018. Organización administrativa y productividad de las ligas deportivas bogotanas. Rev. U.D.C.A Act. \& Div. Cient. 21(2):319-327. https://doi.org/10.31910/rudca.v21.n2.2018.1081

14. SÁNCHEZ, C. 2015. La escuela como proyecto recreativo. Editorial Juan de Castellanos. Tunja, Colombia. 107p.

15. TORRALBA, M.; VIEIRA, M.; LLEIXÁ, T.; GORLA, J. 2014 Evaluación de la coordinación motora en Educación Primaria de Barcelona y Provincia. Rev. Int. Med. y Ciencias de la Act. Física y el Deporte. 16(2):355-371.

16. VIDARTE, J.A.; OROZCO, C.I. 2015. Relaciones entre el desarrollo psicomotor y el rendimiento académico en niños de 5 y 6 años de una institución educativa de la Virginia (Risaralda, Colombia). Rev. Latinoamericana de Estudios Educativos. 11(2):190-204.

17. VIDARTE, C.J.; VÉLEZ, A.C.; PARRA, S.J. 2018. Coordinación motriz e índice de masa corporal en escolares de seis ciudades colombianas. Rev. U.D.C.A Act. \& Div. Cient. 21(1):15-22. https://doi.org/10.31910/rudca.v21.n1.2018.658 